\title{
Antitumor activity of placenta-derived mesenchymal stem cells producing pigment epithelium-derived factor in a mouse melanoma model
}

\author{
QIAOLING CHEN, PING CHENG, NA SONG, TAO YIN, HONG HE, \\ LI YANG, XIANCHENG CHEN and YUQUAN WEI \\ State Key Laboratory of Biotherapy and Cancer Center, West China Hospital, West China Medical School, \\ Sichuan University, Chengdu, Sichuan 610041, P.R. China
}

Received February 22, 2012; Accepted May 24, 2012

DOI: $10.3892 / \mathrm{ol} .2012 .772$

\begin{abstract}
Mesenchymal stem cells (MSCs) are a new tool that can be used for the delivery of therapeutic agents to tumor cells. Among the various types of MSCs, placentaderived MSCs (PDMSCs) have emerged as one of the most attractive vehicles for gene therapy due to their high throughput, lack of ethical concerns, non-invasive procedure for their harvesting and ease of isolation. In this study, we evaluated the antitumor activity of human PDMSCs loaded with recombinant adenoviruses expressing pigment epithelium-derived factor (PEDF). PDMSCs were transduced with adenovirus PEDF and the expression of PEDF was confirmed by western blotting and ELISA. The inhibition of angiogenesis mediated by PEDF-expressing PDMSCs (PDMSC-PEDF) was determined using human umbilical vein endothelial cell (HUVEC) proliferation inhibition assay and migration inhibition assay in vitro. In in vivo experiments, C57BL/6 mice bearing B16-F10 melanoma were treated with intratumoral injection of PDMSC-PEDF twice at a 4-day interval. The tumor volume and weight were recorded. The results demonstrated that the administration of PDMSCPEDF resulted in marked suppression of tumor growth in an established melanoma model, which was associated with a decreased number of microvessels and increased apoptosis of tumor cells compared with the controls. The results suggest that human PDMSCs have potential use as effective delivery vehicles for cancer gene therapy.
\end{abstract}

Correspondence to: Dr Qiaoling Chen, State Key Laboratory of Biotherapy, West China Hospital, Sichuan University, No. 1 Keyuan Road 4, Gaopeng Street, High Technological Development Zone, Chengdu, Sichuan 610041, P.R. China

E-mail: cql166@163.com

Key words: mesenchymal stem cells, pigment epithelium-derived factor, melanoma, gene therapy, anti-angiogenesis

\section{Introduction}

Melanoma is the most aggressive form of skin cancer, and is resistant to the currently used cancer therapeutic modalities (1). Early diagnosis followed by surgical resection improves the prognosis of patients with melanoma. However, despite careful follow-up and treatment with combination chemotherapy or adjuvant therapy, patients frequently develop both local and distant metastases. Patients with distant metastases almost always have a poor clinical outcome (2).

Angiogenesis has a key role in the process of growth and metastasis of primary solid tumors. A tumor usually begins small and is localized, due to the lack of a vascular supply. Thus, depriving a tumor of its vascular supply by means of antiangiogenic agents has been of great interest since its proposal in the 1970s (3). Pigment epithelium-derived factor (PEDF), a $50-\mathrm{kDa}$ secreted glycoprotein from the serine protease inhibitor superfamily, was described as the most potent endogenous inhibitor of angiogenesis (4). PEDF exerts its anti-angiogenic activity by inducing apoptosis in endothelial cells as well as by inhibiting endothelial cell proliferation and migration even in the presence of VEGF (5). The potential of PEDF as a purified protein or using gene transfer approaches with viral and non-viral vectors has been tested in several tumor models including melanoma in previous studies. However, a suboptimal half-life in plasma or its side effects reduce its possible therapeutic effects $(6,7)$. Thus, a more efficient and safer approach is required.

Mesenchymal stem cells (MSCs), which have the potential to differentiate along osteogenic, adipogenic and chondrogenic lineages, were described as novel and efficient therapeutic tools for the targeted delivery and local production of biological agents in tumors (8). The most significant source of mesenchymal stem cells is currently bone marrow. However, cells from the bone marrow may only be obtained through an invasive procedure, and stem cell numbers decrease significantly with the age of the individual (9). For this reason, alternative sources from where MSCs may be isolated have been sought. One significant source is the placenta (10). Several studies indicate that placenta-derived MSCs (PDMSCs) are similar to stem cells from the bone marrow with respect to their 
cell characteristics and multilineage differentiation potential (11-13). The placenta fulfills two main desiderata of cell therapy: obtaining as high as possible number of cells and use of non-invasive methods for their harvesting (14). Moreover, since placenta-derived multipotent cells are fetal in origin, they may generate less of an immune response than adult bone marrow MSCs (15). These characteristics make PDMSCs potential candidates for clinical application in cell-based therapies.

In this study, we evaluated the antitumor activity of human PDMSC transduced with a recombinant adenovirus expressing PEDF in a mouse melanoma model. The results demonstrated that treatment with PEDF-secreting PDMSCs (PDMSC-PEDF) led to a notable inhibition of tumor growth associated with a decreased number of microvessels and an increased apoptotic index of tumor cells.

\section{Materials and methods}

Cell lines and culture. B16-F10 mouse melanoma cell lines and human embryonic kidney 293 cell lines were purchased from the American Type Culture Collection (ATCC, Rockville, MD, USA). Cells were cultured in Dulbecco's modified Eagle's medium (DMEM; Gibco BRL, Grand Island, NY, USA) supplemented with $10 \%$ heat-inactivated fetal bovine serum (FBS; Gibco, Auckland, NZ), 2 mM L-glutamine and $100 \mu \mathrm{g} / \mathrm{ml}$ amikacin. Human umbilical vein endothelial cells (HUVECs) were isolated from human umbilical cord veins as previously described (16), and grown in EBM-2 medium with SingleQuots (Lonza Cologne GmbH, Walkersville, MA, USA) containing VEGF and other growth factors. HUVECs were used between passages 2 and 6 .

Isolation, expansion and characterization of human placentaderived MSCs. After receiving informed consent, placenta was obtained by vaginal delivery or caesarean sections from women following uncomplicated full-term pregnancies. The MSCs were isolated from the placenta as described previously (13). Briefly, placental tissue was dissected following the drainage of umbilical cord blood. Following mechanical and enzymatic treatment, the homogenate was cultured in low-glucose DMEM (Gibco) supplemented with 10\% FBS, $50 \mathrm{U} / \mathrm{ml}$ penicillin and $50 \mu \mathrm{g} / \mathrm{ml}$ streptomycin, and incubated at $37^{\circ} \mathrm{C}$ in a $5 \% \mathrm{CO}_{2}$ atmosphere. After 48 days, the nonadherent hematopoietic cells were discarded and the adherent MSCs were preserved for further expansion. Medium changes were performed twice per week. PDMSCs between passages 5 and 8 were used in the experiments. Phenotype characteristics of the PDMSCs were analyzed by flow cytometry (BD Biosciences, San Jose, CA, USA) using CD34, CD44, CD45, CD73, CD90 and CD105 (BD Biosciences).

Adenoviral transduction of PDMSCs. The adenoviruses were created using the AdEasy system. The viruses were amplified in HEK293 cells and purified on $\mathrm{CsCl}$ gradients according to standard methods (7). The PDMSCs were transduced with recombinant adenovirus at a multiplicity of infection (MOI) of 1500. Prior to transduction, the growth medium was removed and the cells washed once with serum-free medium. Virus infection was performed for $4 \mathrm{~h}$ at $37^{\circ} \mathrm{C}$ and the infection medium was replaced with complete medium. PDMSCs were also infected with adenovirus LacZ (Ad-LacZ) at an MOI of 1500 as a control. After 48 h the virus-infected PDMSCs were harvested for subsequent experiments.

Western blot analysis and ELISA assay. Western blot analysis was conducted as previously described (17). Briefly, PDMSCs were transduced with adenoviruses for $4 \mathrm{~h}$ and the viruscontaining medium was changed for serum-free low-glucose DMEM. After a further $48 \mathrm{~h}$ of incubation, the conditioned media (CM) were collected. The CM were concentrated by super filter (10 kDa, Millipore, Billerica, MA, USA), and western blot assay was performed using a mouse anti-human PEDF monoclonal antibody (R\&D Systems, Boston, MA, USA). The concentration of the PEDF secreted in the CM was measured using a sandwich enzyme-linked immunosorbent assay (ELISA) kit for the human PEDF protein (GBD, San Diego, CA, USA) following the manufacturer's instructions.

HUVEC migration inhibition assay. The Transwell migration assay was used to determine the effect of PEDF secreted from Ad-PEDF-infected PDMSCs on HUVECs and was performed as previously described $(18,19)$. Briefly, HUVECs $\left(2 \times 10^{4}\right.$ per well) were suspended in $200 \mu \mathrm{l}$ of the CM derived from PDMSCs, PDMSC-LacZ and PDMSC-PEDF, respectively, and seeded in the upper chamber which was coated with $50 \mu 1$ Matrigel. The lower well of the Transwell plate was filled with $600 \mu 1$ EBM-2 medium containing various growth factors. After $24 \mathrm{~h}$ of incubation, non-migrated cells were scraped. The cells that had migrated to the opposite side of the membrane were fixed with $100 \%$ methanol, stained with $0.05 \%$ crystal violet, sealed on slides, and counted by microscopy (Olympus; magnification, x100) with 5 fields.

HUVEC proliferation inhibition assay. Anti-angiogenic activity of PEDF produced by PDMSC-PEDF was also confirmed by a HUVEC proliferation inhibition assay as described previously (20). The CM were obtained from PDMSCs, PDMSC-LacZ and PDMSC-PEDF, respectively. HUVECs $\left(8 \times 10^{3}\right)$ had been seeded on 24 -well plates the previous day. At $50 \%$ confluence, the cells were washed with phosphate-buffered saline (PBS) following the removal of the media, and then $500 \mu \mathrm{l} \mathrm{CM}$ was added. The cells were incubated at $37^{\circ} \mathrm{C}$ in $5 \% \mathrm{CO}_{2}$ for $72 \mathrm{~h}$. The cells were then trypsinized, and the number of viable cells was counted using a trypan blue assay.

In vivo experiments. Female C57BL/6 mice, 6 to 8 weeks old, were purchased from the West China Experimental Animal Center of Sichuan University, China, and were maintained in pathogen-free conditions with sterile chow. All animal procedures were conducted according to guidelines provided by the Animal Care and Use Committee of West China Hospital Cancer Center. B16-F10 melanoma cells $\left(1 \times 10^{5}\right)$ were injected into the right flank of each mouse subcutaneously. When tumor diameters reached $3 \mathrm{~mm}$, mice were randomly divided into four groups: i) mice treated with $\mathrm{PBS}$, ii) mice treated with $5 \times 10^{5}$ PDMSCs, iii) mice treated with $5 \times 10^{5}$ PDMSCLacZ, and iv) mice treated with $5 \times 10^{5}$ PDMSC-PEDF. The tumors were treated twice by intratumoral injection at a 4-day 
interval. Tumor growth was monitored every 3 days by caliper and the volume was calculated as $0.52 \mathrm{x}$ length $\mathrm{x}$ width ${ }^{2}(21)$. When any mice began to moribund they were sacrificed. Subcutaneous tumors from sacrificed mice were removed and the weight was recorded.

Immunohistochemical analysis and TUNEL assay. To determine the effect of anti-angiogenesis treatment on vessel density, frozen sections were fixed in acetone, incubated and probed with an anti-CD31 antibody (BD Biosciences) as previously described (22). The sections were then visualized and microvessels were calculated with a microscope (Olympus) at a magnification of $x 400$.

The analysis of apoptotic cells in tumor tissue was performed by terminal deoxynucleotidyl transferase-mediated dUTP nick-end labeling (TUNEL) staining using the DeadEnd Fluorometric TUNEL system (Promega, Madison, WI, USA) following the manufacturer's guide. Images of the sections were captured using a fluorescence microscope (Olympus). The apoptotic index was calculated by dividing the number of TUNEL-positive cells by the total number of cells in the field (5 high-power fields per slide).

Statistical analysis. Values were shown as the means \pm SEM (standard error of the mean), and SPSS 17.0 was used for statistical analysis. The statistical significances among the different groups were evaluated using one-way analysis of variance (ANOVA). $\mathrm{P}<0.05$ was considered to indicate a statistically significant result.

\section{Results}

Adenoviral transduction of PDMSCs and confirmation of $P E D F$ expression in vitro. The phenotype characteristics of the isolated and expanded PDMSCs were confirmed by flow cytometry. PDMSCs were cultured to reach to $\sim 90 \%$ confluence and incubated with adenoviruses at a MOI of 1500 for $4 \mathrm{~h}$. After $48 \mathrm{~h}$, the secreted PEDF in the CM was confirmed by western blot and ELISA. Western blot showed that PEDF was only detected in the CM from Ad-PEDF-transduced PDMSCs, but not in Ad-LacZ-transduced PDMSCs nor in untransduced PDMSCs (Fig. 1A). These results indicate that our recombinant adenovirus successfully transferred the PEDF gene into PDMSCs and produced secretory protein. ELISA revealed that PDMSC-PEDF cells had secreted PEDF into the $\mathrm{CM}$ at a concentration of $65.2 \pm 4.9 \mathrm{ng} / \mathrm{ml}$; however, only a minimal amount of PEDF was detected in the CM from Ad-LacZ-transduced and untransduced PDMSCs (Fig. 1B).

PEDF from PDMSC-PEDF inhibited the migration and proliferation of HUVECs in vitro. The bioactivity of PEDF expressed by PDMSC-PEDF was verified by HUVEC migration inhibition assay and proliferation inhibition assay. The CM from PDMSC-PEDF markedly reduced endothelial cell migration, but the control CM from Ad-LacZ-transduced and untransduced PDMSCs had no inhibitory effect on it $(\mathrm{P}<0.05)$ (Fig. 2A and B). The CM from PDMSCs-PEDF significantly inhibited HUVEC proliferation compared with that from PDMSCs or PDMSC-LacZ $(\mathrm{P}<0.05)$ (Fig. 2C). These results indicate that the secretory PEDF was functional.
$\mathbf{A}$

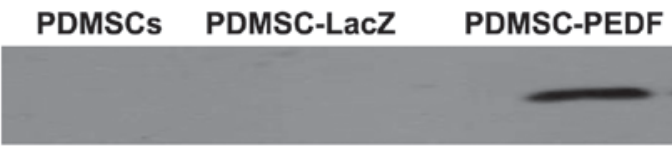

B

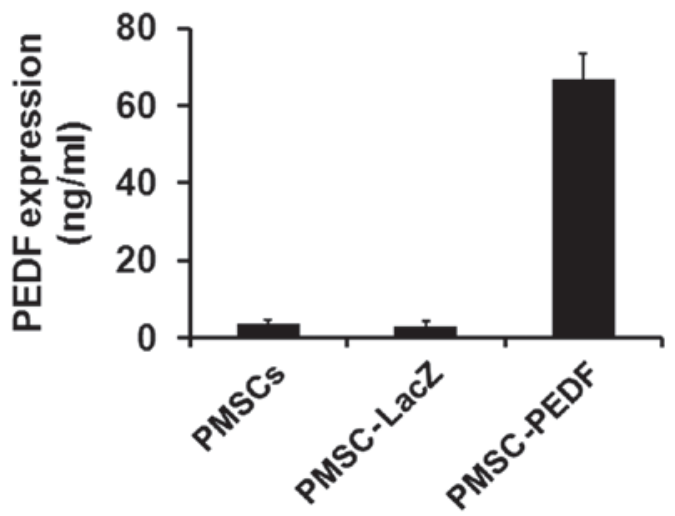

Figure 1. Verification of PEDF secreted by PDMSC-PEDF. (A) The conditioned media (CM) derived from Ad-PEDF-infected PDMSCs were detected by antibodies reactive to human PEDF, but results from the western blot analysis showed negative staining in CM derived from the control PDMSCs and PDMSC-LacZ. (B) PEDF concentration in the CM derived from untransduced PDMSCs, PDMSC-LacZ and PDMSC-PEDF was measured by enzyme-linked immunosorbent assay. PDMSC, placenta-derived mesenchymal stem cells; PEDF, pigment epithelium-derived factor.

PDMSC-PEDF inhibited the growth of B16-F10 melanoma in vivo. To examine the therapeutic effect of PEDF genemodified PDMSCs in vivo, C57BL/6 mice bearing B16-F10 subcutaneous tumors were treated with PBS, 5x10 5 PDSCs, $5 \times 10^{5}$ PDMSC-LacZ, or $5 \times 10^{5}$ PDMSC-PEDF two times at a 4-day interval by intratumoral injection. The tumor volume in the PDMSC-PEDF-treated group was significantly smaller than that in the control groups $(\mathrm{P}<0.05)$. The mean tumor volume $( \pm \mathrm{SD})$ in PDMSC-PEDF-treated mice was $1287.1 \pm 284.3 \mathrm{~mm}^{3}$ versus $3439.1 \pm 417 \mathrm{~mm}^{3}$ in PDMSCs-LacZtreated mice, $3620.4 \pm 279.7 \mathrm{~mm}^{3}$ in PDMSC-treated mice and $3782.4 \pm 315.3 \mathrm{~mm}^{3}$ in PBS-treated mice (Fig. 3A). There was no significant difference between the PDMSC-treated group and the PBS-treated group ( $\mathrm{P}>0.05)$. The tumor weight was measured when the mice were sacrificed. The mean tumor weights were $2.89 \pm 0.19,2.76 \pm 0.41,2.56 \pm 0.42$ and $0.98 \pm 0.13 \mathrm{~g}$ in the PBS-, PDMSC-, PDMSC-LacZ- and PDMSC-PEDFtreated groups, respectively (Fig. 3B). Taken together, the data demonstrate that PDMSC-PEDF has a significant and prolonged inhibitory effect on the tumor growth of B16-F10 melanoma in vivo.

PDMSC-PEDF inhibited angiogenesis and induced apoptosis in vivo. Angiogenesis within the tumor tissue was estimated by counting the number of microvessels on the section stained with an anti-CD31 antibody (Fig. 4A). The microvessel density was significantly reduced in the PDMSC-PEDF-treated group compared with the other groups $(\mathrm{P}<0.05)$ (Fig. 4C). Apoptotic cells in tumors were determined by TUNEL assay (Fig. 4B). The number of apoptotic cells in the PDMSC-PEDF-treated group was found to be significantly higher than that of the other groups $(\mathrm{P}<0.05)$ (Fig. 4D). 
A
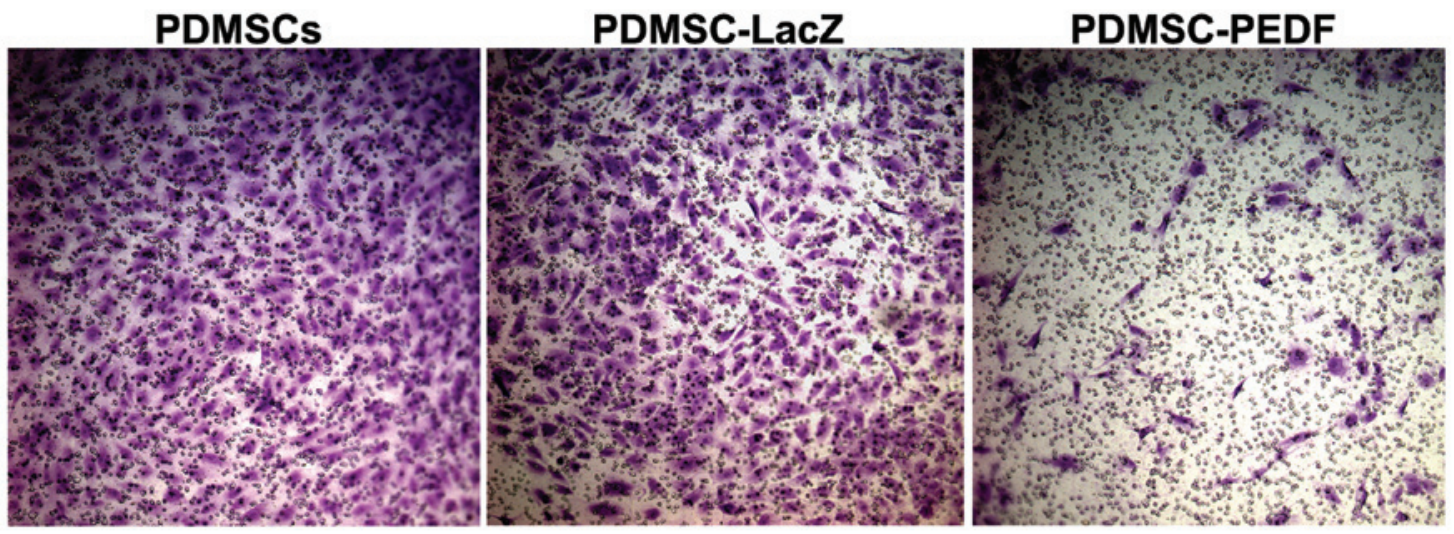

B



C

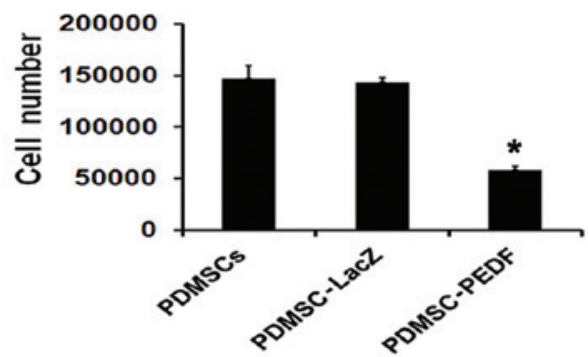

Figure 2. Anti-angiogenic activity of PEDF secreted by PDMSC-PEDF in vitro. (A) PEDF produced by PDMSC-PEDF inhibited HUVEC migration in Transwell assay (magnification, $x 100$ ). (B) The number of migrated cells was counted in each group ( $\mathrm{P}<0.05$ ). (C) PEDF produced by PDMSC-PEDF markedly inhibited HUVEC proliferation compared with the PDMSC and PDMSC-LacZ groups ("P<0.05). PDMSC, placenta-derived mesenchymal stem cells; PEDF, pigment epithelium-derived factor.

A

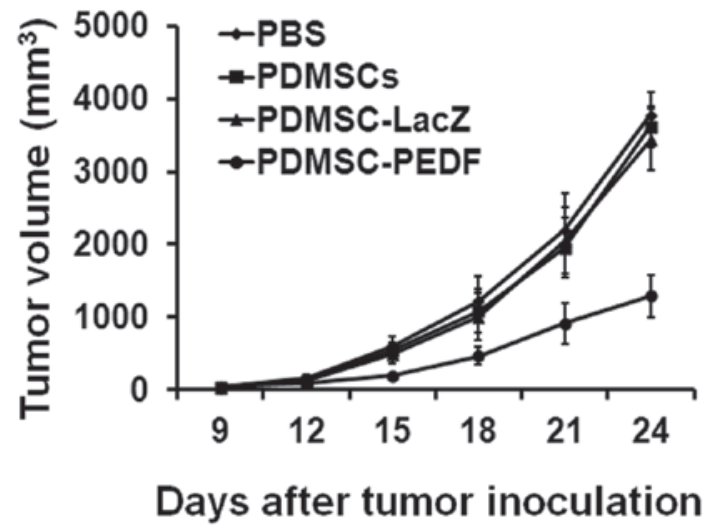

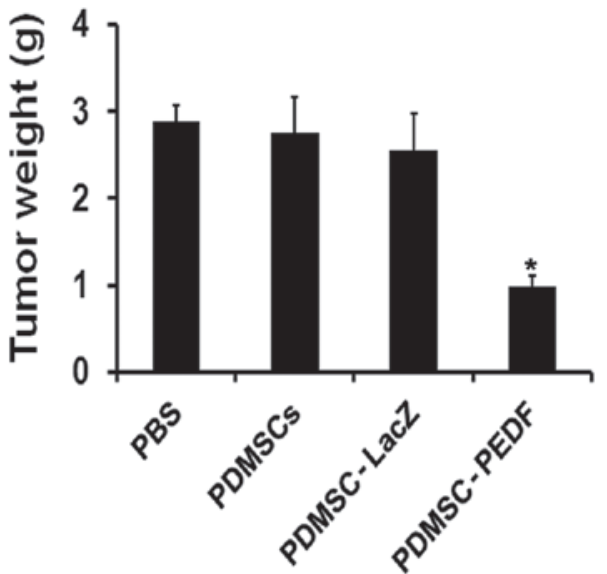

Figure 3. Antitumor efficacy of PDMSC-PEDF in vivo. Tumor-bearing female C57BL/6 mice were treated intratumorally with $100 \mu 1$ PBS, 5x10 $\mathrm{PDMSCs}$, $5 \times 10^{5}$ PDMSC-LacZ or $5 \times 10^{5}$ PDMSC-PEDF twice at 4-day intervals. (A) A significant decrease in tumor volume was observed in PDMSC-PEDF-treated mice, compared with the controls $(\mathrm{P}<0.05)$. (B) Comparison of the tumor weight. Significant differences between the PDMSC-PEDF group and control groups are shown ("P<0.05). PDMSC, placenta-derived mesenchymal stem cells; PEDF, pigment epithelium-derived factor.

\section{Discussion}

In this study, we focused on the possibility of employing human xenogeneic PDMSCs as a vehicle for the delivery of PEDF to mouse B16-F10 melanoma. The data showed that treatment with PDMSCs expressing PEDF led to a considerable reduction of tumor growth compared with the control groups.

For tumors to develop, grow and spread, angiogenesis is the primary mechanism involved, whereby new blood vessels form from preexisting ones (23). Melanoma has been well-documented as an angiogenic tumor type, clearly demonstrating new vessel formation as an essential step in disease progression from atypical melanocytes, through radial growth to the aggressive vertical growth phase. Thus, anti-angiogenic therapy has been considered to be a new direction to fight melanoma (24). PEDF was described as the most potent endogenous inhibitor of angiogenesis and is capable of inducing apoptosis in endothelial cells as well as inhibiting endothelial 

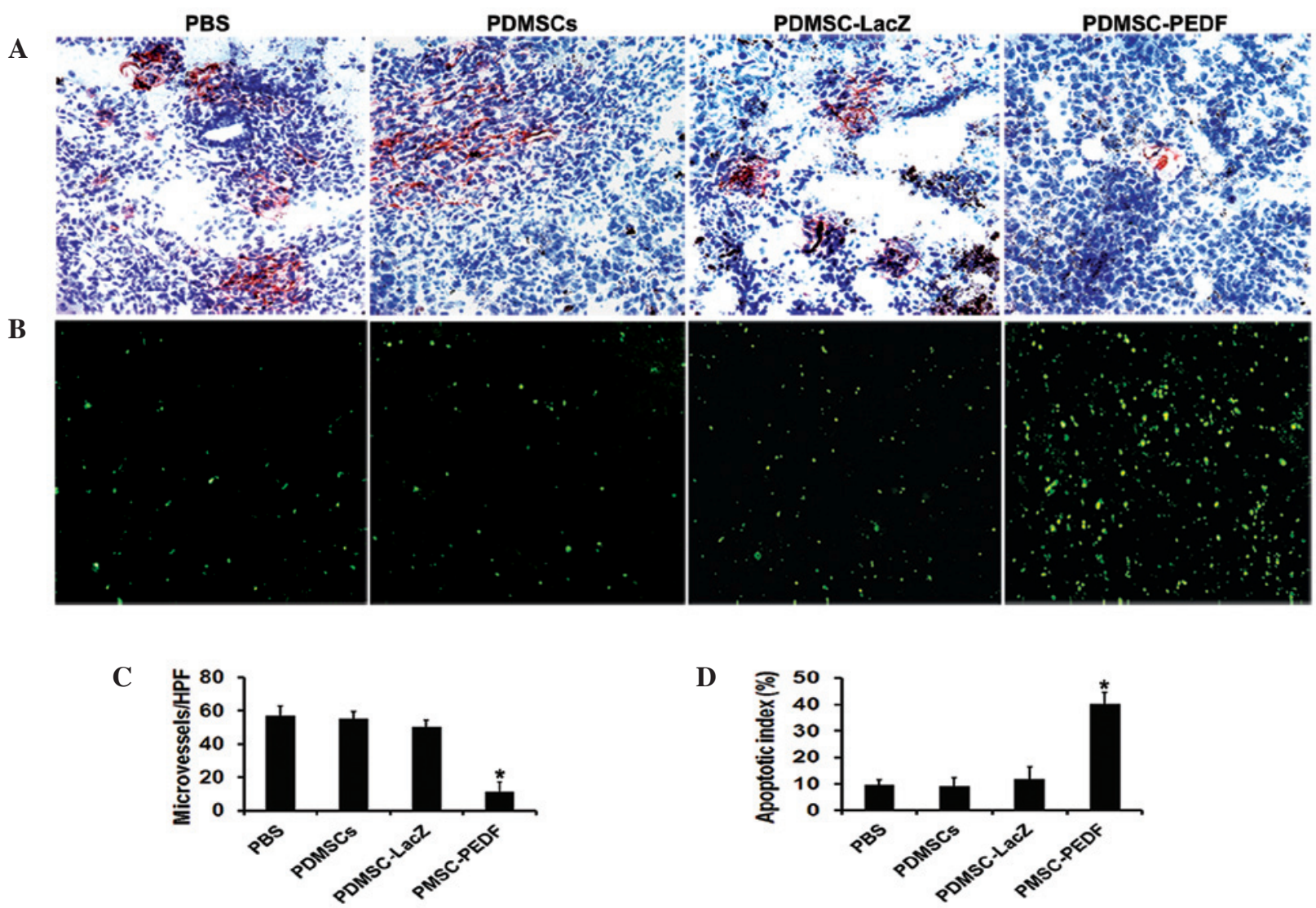

D



Figure 4.CD31 staining and TUNEL assay in tumor tissue.(A) Representative tumor sections stained with an anti-CD31 antibody are shown (magnification, x200). (B) Apoptotic cells were identified by TUNEL assay in each group (magnification, x200). (C) Microvessel density (MVD) was determined by counting the number of microvessels per high-power field (HPF) in the sections. The PDMSC-PEDF group showed a marked decrease in MVD compared to the control groups ( $\mathrm{P}<0.05)$. (D) The percentage of apoptosis was determined by counting the number of apoptotic cells and dividing by the total number of cells in the field. Bottom columns show the ultimate apoptotic indices for tumor tissue from different groups ( $\left.{ }^{*} \mathrm{P}<0.05\right)$.

cell proliferation and migration. A number of studies demonstrate that a low level of PEDF is associated with the increased incidence of metastasis and poor malignancy prognosis in various tumors (25). Garcia et al and Abe et al showed that overexpression of PEDF in malignant melanoma cell lines by stable transfection with retrovirus and plasmids, respectively, markedly reduced intratumoral microvessel density as well as primary tumor growth and metastasis $(26,27)$. In our previous study, the potent antitumor activity of adenovirus-mediated PEDF was demonstrated in B16-F10 melanoma. However, the high immunogenicity of the adenovirus, which induces a major humoral and cellular immune response, results in rapid clearance of the virus as well as side effects such as inflammation in vivo (7). To overcome these problems, we focused on MSC-based gene therapy.

Recently, MSCs have been used as a new therapeutic strategy for the targeted delivery and local production of biological agents in tumors to improve the efficacy and minimize the toxicity. This is because MSCs have tumor-targeting properties, can be easily isolated and expanded to the numbers required for use, and can be genetically manipulated with viral vectors (8). Adult bone marrow (BM) is the common source of MSCs used in clinical settings. However, invasive isolation procedures and low yield for BM-MSCs are an obstacle to their use in cellular therapy (12). As an alternative source, PDMSCs exhibit clear advantages: placenta can be obtained at every delivery and its use does not pose any ethical problems. Furthermore, the recovery of cells from this tissue does not involve any invasive procedures for the donor (28). MSCs derived from placenta have low immunogenicity associated with a lack or low level of expression of MHC class II molecules and co-stimulatory molecules in the same way as bone marrow-derived MSCs (29). Moreover, since placenta-derived cells are fetal in origin, they may generate less of an immune response than BM-MSCs (30). It has been demonstrated that cells isolated from amniotic and chorionic membranes do not induce an allogeneic or xenogeneic immune response in mixed lymphocyte reactions and are capable of actively suppressing the proliferation of lymphocytes in vitro (15). Several studies have already reported a prolonged survival of human placenta-derived cells following xenogeneic transplantation into immunocompetent animals including rats $(31,32)$, swine (31), and bonnet monkeys (33), with no evidence of immunological rejection.

In our study, we demonstrated that PDMSCs may be genetically modified with Ad-PEDF and express high levels of PEDF in vitro. Furthermore, we revealed that the PEDF produced by these engineered PDMSCs is a functional protein with potent inhibitory effects on HUVEC proliferation and migration. In the in vivo study, we found that PDMSC-PEDF efficiently inhibited the growth of B16-F10 melanoma. CD31 staining and TUNEL assay revealed a significant reduction in microvessel density and an increase in the apoptotic index in the tumor 
tissue of the PDMSC-PEDF-treated group. The observed antitumoral effect was a result of the expression of PEDF and not a result of an immune response to PDMSCs, since the injection of control PDMSCs had no effect on tumor growth.

In summary, our investigation demonstrated that adenovirus-mediated anti-angiogenesis gene therapy based on xenogeneic PDMSCs inhibits the growth of B16-F10 melanoma. Thus, the use of PDMSCs as a delivery vehicle of therapeutic genes is likely to be of great interest for the clinical application of stem cell-based cancer therapy. Further studies should be carried out in an allogeneic setting to detect whether PDMSCs survive longer due to their low immunogenicity and exert a more effective antitumor activity.

\section{Acknowledgements}

The authors thank members of the State Key Laboratory of Biotherapy for their helpful discussions. This study was supported by the National Natural Science Foundation of China (30973507) and the National 973 Basic Research Program of China (2010CB529900 and 2010CB529906).

\section{References}

1. Soengas MS and Lowe SW: Apoptosis and melanoma chemoresistance. Oncogene 22: 3138-3151, 2003.

2. Rook G: Tumours and Coley's toxins. Nature 357: 545, 1992.

3. Dass CR, Tran TM and Choong PF: Angiogenesis inhibitors and the need for anti-angiogenic therapeutics. J Dent Res 86: 927-936, 2007.

4. Dawson DW, Volpert OV, Gillis P, et al: Pigment epitheliumderived factor: a potent inhibitor of angiogenesis. Science 285 245-248, 1999.

5. Elayappan B, Ravinarayannan H, Pasha SP, Lee KJ and Gurunathan S: PEDF inhibits VEGF- and EPO- induced angiogenesis in retinal endothelial cells through interruption of PI3K/ Akt phosphorylation. Angiogenesis 12: 313-324, 2009.

6. Doll JA, Stellmach VM, Bouck NP, et al: Pigment epitheliumderived factor regulates the vasculature and mass of the prostate and pancreas. Nat Med 9: 774-780, 2003.

7. Yang LP, Cheng P,Peng XC, et al: Anti-tumor effect of adenovirusmediated gene transfer of pigment epithelium-derived factor on mouse B16-F10 melanoma. J Exp Clin Cancer Res 28: 75, 2009.

8. Fritz V and Jorgensen C: Mesenchymal stem cells: an emerging tool for cancer targeting and therapy. Curr Stem Cell Res Ther 3: 32-42, 2008.

9. Rao MS and Mattson MP: Stem cells and aging: expanding the possibilities. Mech Ageing Dev 122: 713-734, 2001.

10. Pasquinelli G, Tazzari P, Ricci F, et al: Ultrastructural characteristics of human mesenchymal stromal (stem) cells derived from bone marrow and term placenta. Ultrastruct Pathol 31: 23-31, 2007.

11. Rus Ciuca D, Soritau O, Susman S, Pop VI and Mihu CM: Isolation and characterization of chorionic mesenchyal stem cells from the placenta. Rom J Morphol Embryol 52: 803-808, 2011.

12. In 't Anker PS, Scherjon SA, Kleijburg-van der Keur C, et al: Isolation of mesenchymal stem cells of fetal or maternal origin from human placenta. Stem Cells 22: 1338-1345, 2004.

13. Kadam S, Muthyala S, Nair P and Bhonde R: Human placentaderived mesenchymal stem cells and islet-like cell clusters generated from these cells as a novel source for stem cell therapy in diabetes. Rev Diabet Stud 7: 168-182, 2010.
14. Mihu CM, Mihu D, Costin N, Rus Ciuca D, Susman S and Ciortea R: Isolation and characterization of stem cells from the placenta and the umbilical cord. Rom J Morphol Embryol 49: 441-446, 2008

15. Chang CJ, Yen ML, Chen YC, et al: Placenta-derived multipotent cells exhibit immunosuppressive properties that are enhanced in the presence of interferon-gamma. Stem Cells 24: 2466-2477, 2006.

16. Jaffe EA, Nachman RL, Becker CG and Minick CR: Culture of human endothelial cells derived from umbilical veins. Identification by morphologic and immunologic criteria. J Clin Invest 52: 2745-2756, 1973.

17. Liu JY, Wei YQ, Yang L, et al: Immunotherapy of tumors with vaccine based on quail homologous vascular endothelial growth factor receptor-2. Blood 102: 1815-1823, 2003.

18. Cao G, O'Brien CD, Zhou Z, et al: Involvement of human PECAM-1 in angiogenesis and in vitro endothelial cell migration. Am J Physiol Cell Physiol 282: C1181-C1190, 2002.

19. Zhang S, Cao Z, Tian H, et al: SKLB1002, a novel potent inhibitor of VEGF receptor 2 signaling, inhibits angiogenesis and tumor growth in vivo. Clin Cancer Res 17: 4439-4450, 2011.

20. Hu M, Yang JL, Teng H, et al: Anti-angiogenesis therapy based on the bone marrow-derived stromal cells genetically engineered to express sFlt-1 in mouse tumor model. BMC Cancer 8: 306, 2008.

21. Wang L, Schmitz V, Perez-Mediavilla A, Izal I, Prieto J and Qian C: Suppression of angiogenesis and tumor growth by adenoviral-mediated gene transfer of pigment epithelium-derived factor. Mol Ther 8: 72-79, 2003.

22. Peng XC, Yang L, Yang LP, et al: Efficient inhibition of murine breast cancer growth and metastasis by gene transferred mouse survivin Thr34-->Ala mutant. J Exp Clin Cancer Res 27: 46, 2008.

23. North S, Moenner M and Bikfalvi A: Recent developments in the regulation of the angiogenic switch by cellular stress factors in tumors. Cancer Lett 218: 1-14, 2005.

24. Emmett MS, Dewing D and Pritchard-Jones RO: Angiogenesis and melanoma - from basic science to clinical trials. Am J Cancer Res 1: 852-868, 2011.

25. Ek ET, Dass CR and Choong PF: Pigment epithelium-derived factor: a multimodal tumor inhibitor. Mol Cancer Ther 5: 1641-1646, 2006.

26. Garcia M, Fernandez-Garcia NI, Rivas V, et al: Inhibition of xenografted human melanoma growth and prevention of metastasis development by dual antiangiogenic/antitumor activities of pigment epithelium-derived factor. Cancer Res 64: 5632-5642, 2004.

27. Abe R, Shimizu T, Yamagishi S, et al: Overexpression of pigment epithelium-derived factor decreases angiogenesis and inhibits the growth of human malignant melanoma cells in vivo. Am J Pathol 164: 1225-1232, 2004.

28. Evangelista M, Soncini M and Parolini O: Placenta-derived stem cells: new hope for cell therapy? Cytotechnology 58: 33-42, 2008.

29. Parolini O, Alviano F, Bagnara GP, et al: Concise review: isolation and characterization of cells from human term placenta: outcome of the first international Workshop on Placenta Derived Stem Cells. Stem Cells 26: 300-311, 2008.

30. Wagner JE, Rosenthal J, Sweetman R, et al: Successful transplantation of HLA-matched and HLA-mismatched umbilical cord blood from unrelated donors: analysis of engraftment and acute graft-versus-host disease. Blood 88: 795-802, 1996.

31. Bailo M, Soncini M, Vertua E, et al: Engraftment potential of human amnion and chorion cells derived from term placenta. Transplantation 78: 1439-1448, 2004.

32. Meng XT, Li C, Dong ZY, et al: Co-transplantation of bFGFexpressing amniotic epithelial cells and neural stem cells promotes functional recovery in spinal cord-injured rats. Cell Biol Int 32: 1546-1558, 2008

33. Sankar V and Muthusamy R: Role of human amniotic epithelial cell transplantation in spinal cord injury repair research. Neuroscience 118: 11-17, 2003. 